\title{
Bile Reflux Scintigraphy After Mini-Gastric Bypass
}

\section{Saarinen, Tuure}

2017-08

Saarinen , T , Räsänen , J V , Salo , J , Loimaala , A, Pitkonen , M , Leivonen, M \& Juuti , A 2017 , ' Bile Reflux Scintigraphy After Mini-Gastric Bypass ' , Obesity Surgery , vol. 27 , no. 8 , pp. 2083-2089 . https://doi.org/10.1007/s11695-017-2608-7

http://hdl.handle.net/10138/238192

https://doi.org/10.1007/s11695-017-2608-7

publishedVersion

Downloaded from Helda, University of Helsinki institutional repository.

This is an electronic reprint of the original article.

This reprint may differ from the original in pagination and typographic detail.

Please cite the original version. 


\title{
Bile Reflux Scintigraphy After Mini-Gastric Bypass
}

\author{
Tuure Saarinen ${ }^{1,2}$ (1) • Jari Räsänen ${ }^{3} \cdot$ Jarmo Salo $^{3}$ - Antti Loimaala ${ }^{4} \cdot$ Miia Pitkonen $^{4}$ • \\ Marja Leivonen ${ }^{5} \cdot$ Anne Juuti $^{1}$
}

Published online: 18 February 2017

(C) Springer Science+Business Media New York 2017

\begin{abstract}
Background Significant weight-loss and diabetes remission have been reported after mini-gastric bypass (MGB). Concern has been raised regarding postoperative bile reflux (BR), but it has not been demonstrated in previous studies. We set out to find out if BR is evident in hepatobiliary scintigraphy after MGB.

Methods Nine consecutive patients, seven with type 2 diabetes, underwent MGB ( $15 \mathrm{~cm}$ gastric tube, $250-275 \mathrm{~cm}$ biliary limb) at our institution with a 12-month follow-up, with none lost to follow-up. Then, 10.7 months (8.6-13.0) after MGB, all patients underwent hepatobiliary scintigraphy and a reflux symptom questionnaire (GerdQ) was filled out. A gastroscopy with biopsies was done for all patients with a bile-refluxpositive scintigraphy.

Results Mean age at operation was 56 years (41-65) and preoperative BMI $43.1 \mathrm{~kg} / \mathrm{m}^{2}$ (34.2-54.6). Mean \%EWL was $83.9(49.5-128.3)$ at 12 months. Four patients reached diabetes remission and two became insulin-independent. Hepatobiliary scintigraphy showed a transient BR into the
\end{abstract}

Tuure Saarinen

tuure.saarinen@hus.fi

Jari Räsänen

jari.rasanen@hus.fi

Jarmo Salo

jarmo.salo@hus.fi

Antti Loimaala

antti.loimaala@hus.fi

Miia Pitkonen

miia.pitkonen@hus.fi

Marja Leivonen

marja.leivonen@epshp.fi gastric tube for five patients. Bile tracer was found in the gastric tube at $23-58 \mathrm{~min}$ after the tracer injection and highest activity was $8 \%(1-8 \%)$ at $58 \mathrm{~min}$. Bile tracer was not found in the esophagus of any of the patients. One patient with a positive scintigraphy in the gastric tube required re-operation. Two patients with reflux symptoms had a negative scintigraphy.

Conclusion Our results indicate that transient bile reflux is common after MGB in the gastric tube, but not in the esophagus. The clinical relevance of bile reflux needs further studies.

Keywords Mini-gastric bypass · Single-anastomosis gastric bypass $\cdot$ Bariatric surgery $\cdot$ Bile reflux $\cdot$ Scintigraphy

\section{Introduction}

Laparoscopic mini-gastric bypass (MGB) was developed from Mason's loop gastric bypass in 1997 [1]. In recent years,

\author{
Anne Juuti \\ anne.juuti@hus.fi
}

Department of Abdominal Surgery, Helsinki University Hospital, Haartmaninkatu 4, 00029 Helsinki, HUS, Finland

2 Faculty of Medicine, University of Helsinki, Helsinki, Finland

3 Department of General Thoracic and Esophageal Surgery, Helsinki University Hospital, Helsinki, Finland

4 Department of Nuclear Medicine, Helsinki University Hospital, Helsinki, Finland

5 Department of Surgery, Central Hospital of Seinäjoki, Seinäjoki, Finland 
MGB has become increasingly popular among bariatric surgeons and is nowadays performed all over the world. Since the first report by Rutledge et al. in 2001, many reports have shown good results with MGB regarding weight-loss and effect on associated diseases, especially type 2 diabetes [1-4]. It has also been stated that MGB is faster and less prone to surgical complications compared to the traditional Roux-enY gastric bypass (RYGB) [3, 4]. However, concern has been raised regarding complications related to MGB procedure, in particular reflux of jejunal contents including bile and pancreatic juices into the gastric tube or even esophagus, in the literature also referred to as bile reflux (BR) or duodenogastric reflux $[5,6]$. Studies on hepatobiliary scintigraphies, impedance, or intragastric bile measurements after MGB have previously not been published, that could reveal the presence of bile in the gastric tube or esophagus. Hepatobiliary scintigraphy is a non-invasive and physiologic method for the detection of bile reflux [7, 8]. It has been shown to be more sensitive and specific compared to gastric juice examinations in the detection of BR [9].

We started performing MGB in December 2014 in Helsinki University Hospital. We set out to investigate, whether we can demonstrate BR after MGB by using dynamic SPECTimaging with a non-invasive hepatobiliary scintigraphy using ${ }^{99 \mathrm{~m}} \mathrm{Tc}$-labeled mebrofenin.

\section{Methods}

\section{Patients}

Nine out of 13 patients, who underwent MGB at our institution between December 2014 and April 2015, were willing to participate in this study. Bariatric surgery was indicated according to international guidelines [10]. Prior to surgery, Helicobacter pylori was tested and eradicated. All patients were prescribed multivitamin and calcium supplements after the operation. All patients had follow-up visits with extensive blood work including glycohemoglobin ( $\mathrm{HbA1C}$ ), calcium, iron, and albumin at 1, 3, 6, and 12 months after the surgery. In addition to standard visits, all patients were invited for a hepatobiliary scintigraphy 8 to 13 months after the surgery when patients had been able to lose most of their excess weight. Prior to the scintigraphy, all patients filled out a GerdQ questionnaire, which is validated for detection of reflux symptoms [11]. All patients, who had signs of BR in scintigraphy, were also invited for an additional gastroscopy with biopsies. Surgical ethical committee of Helsinki University Hospital approved the study design and informed consent was obtained from all individual participants included in the study. Weight-loss is reported as excess weight-loss (\%EWL), calculated from the weight at preoperative visit. Numeric values are reported as mean (min-max).

\section{Surgical Technique}

Our technique is essentially similar to the MGB technique by Rutledge [1]. We used a long omega loop in order to achieve marked effect on diabetes as well as weight-loss. All operations were performed under general anesthesia using a standard 5-port laparoscopy. After carbon dioxide insufflation, a 15-cm-long gastric tube was divided along the lesser curvature starting at the crow's foot with Endo-GIA ${ }^{\circledR}$ (Medtronic, Minneapolis, MN, USA) staplers and calibrated with a 40Fr bougie. A jejunal loop was lifted $250-275 \mathrm{~cm}$ from the ligament of Treitz and anastomosed antecolic to the gastric tube with an endo-GIA stapler. The remaining anastomotic defect was sewn with a running 3-0 Vicryl (Ethicon, Somerville, New Jersey, USA) suture intra-abdominally. Anastomosis was tested with methylene blue and the Petersen defect was closed for all patients. No abdominal drainage, urinary catheter, nor naso-gastric tube were left in place for any of the patients. All patients were mobilized and received oral liquids within $2 \mathrm{~h}$ after the operation.

\section{Hepatobiliary Scintigraphy}

Mebrofenin labeled with ${ }^{99 \mathrm{~m}}$ Technethium (Bridatec, GE Healthcare Life Sciences Core Imaging, Pittsburgh, PA, USA) was used as bile tracer after a 12-h fasting period. Intravenously injected mebrofenin is cleared by the liver and conjugated to bile. Thus, bile flow through the intestine can be observed by detecting the fate of the tracer [12]. Imaging consisted of dynamic gamma camera (Symbia T2 System; Siemens, Munich, Germany) imaging with a low-energy collimator, a static image, and single-photon emission computed tomography/computed tomography (SPECT-CT). Dynamic imaging (72 frames $\times 50 \mathrm{~s}$ ) began from the ${ }^{99 \mathrm{~m}} \mathrm{Tc}$-mebrofenin injection and continued for $60 \mathrm{~min}$. Tracer injection (190 MBq) was given in supine position into cubital vein with a gamma camera detector above the patients' abdomen. A 10 min static image and SPECT-CT was taken $2 \mathrm{~h}$ after tracer injection in order to localize BR in the esophagus or the gastric tube. SPECT was performed by acquiring 60 projections $(20 \mathrm{~s}$ per projection) over $180^{\circ}$ on a non-circular imaging mode.

A nuclear medicine physician first estimated the dynamic data. If BR was detected, a region of interest (ROI) was placed into that area. The onset of BR, its duration, and activity were defined from the time activity curves. The amount of duodenogastric bile reflux (BRi) was calculated as ratio of total counts in the first frame of the dynamic series in the liver to maximum activity in the ROI. Fatty meal stimulation for gallbladder emptying was not used in this study.

\section{Endoscopic and Histological Assessment}

Four patients with a BR-positive scintigraphy were invited for a gastroscopy with biopsies. Gastroscopies were performed 
Table 1 Patient demographics and weight-loss during the follow-up

\begin{tabular}{llllll}
\hline & Gender & Age (y) & \multicolumn{2}{c}{ BMI $\left(\mathrm{kg} / \mathrm{m}^{2}\right)$} & \multirow{2}{*}{$\%$ EWL } \\
\cline { 4 - 5 } & & & Preop & 12 mo & 12 mo \\
\hline Patient 1 & F & 51 & 37.3 & 26.7 & 88.2 \\
Patient 2 & M & 64 & 45 & 33 & 76.3 \\
Patient 3 & M & 51 & 50.9 & 38 & 49.5 \\
Patient 4 & M & 55 & 40.7 & 26.2 & 92.3 \\
Patient 5 & M & 65 & 35.3 & 27 & 78.0 \\
Patient 6 & F & 56 & 52.8 & 29.6 & 73.6 \\
Patient 7 & M & 41 & 34.6 & 22.7 & 128.3 \\
Patient 8 & F & 64 & 37.8 & 23.9 & 109.0 \\
Patient 9 & F & 59 & 48.4 & 34.29 & 60.3 \\
\hline
\end{tabular}

$y$ years, mo months, $B M I$ body mass index, $\% E W L$ excess weight-loss, preop prior to mini-gastric bypass

without sedation with a flexible endoscope (Olympus Q190, Tokyo, Japan) and mucosal biopsies were obtained from the gastric tube, cardia, and esophagus. Biopsy specimen was prepared with serial sections ( $5 \mu \mathrm{m}$ thick) from formalinfixed and paraffin-embedded biopsy specimen after hematoxylin-eosin (HE) and AB-PAS-staining.

\section{Reflux Symptom Questionnaire (GerdQ)}

GerdQ questionnaire was developed as a clinical tool for evaluation reflux symptoms [11].The original questionnaire is in
English, but the patients in our series are native speakers of either Finnish or Swedish. We used a previously validated Swedish version of the questionnaire and we translated the questionnaire into Finnish according to a validated translation procedure in which two native speakers of Finnish translated the questionnaire from English to Finnish. A third native speaker of Finnish created a reconciled version which was translated back to English by a native speaker of Finnish and English. The back translation was essentially identical to the original version and therefore the interim version was accepted for pilot testing with ten patients with gastrointestinal reflux disease. After pilot testing, the final translation was approved by consensus of all parties involved.

\section{Results}

Nine patients (five men) underwent MGB between December 2014 and April 2015. Mean age at operation was 56.2 years (41-65). Mean preoperative BMI was $43.1 \mathrm{~kg} / \mathrm{m}^{2}$ (34.254.6). Mean \%EWL was $25.4 \%$ (12.0-34.8), 51.5\% (29.4 $72.8)$, and $70.1 \%(41.2-108.7)$ at 1,3 , and 6 months and $83.9 \%$ (49.5-128.3) at 12 months, respectively (Table 1). Prior to surgery, seven patients had type 2 diabetes for 12.9 years (5.0-30.0) and four of them were insulin-dependent. During the follow-up, four patients reached a complete remission of diabetes and two patients became independent of insulin. Resolution of high blood pressure and dyslipidemia was observed in three and two patients, respectively (Table 2).
Table 2 Characterization of obesity-related conditions

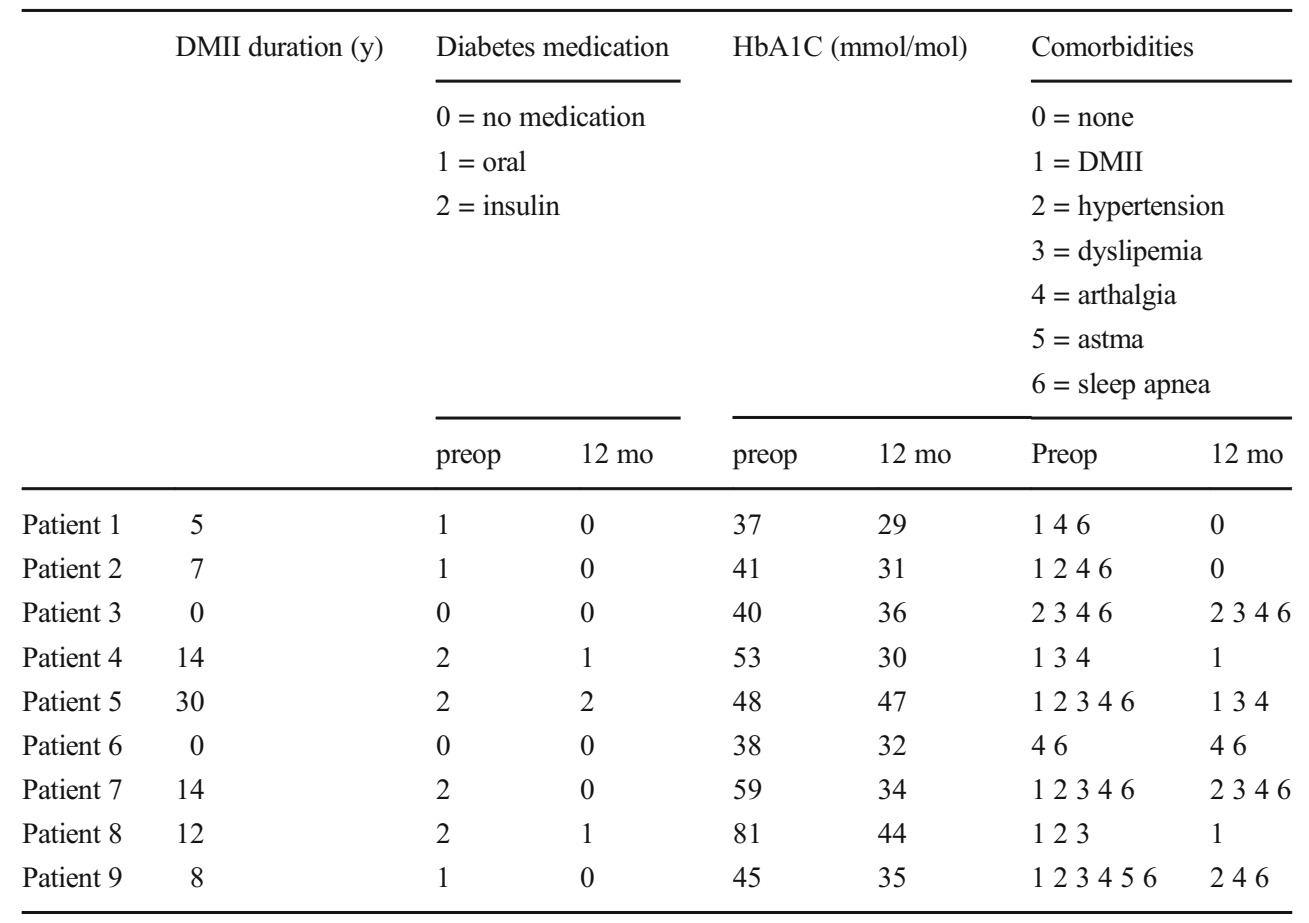

DMII type 2 diabetes mellitus, $y$ years, mo months, preop prior to mini-gastric bypass, $H b A 1 C$ glycohemoglobin 


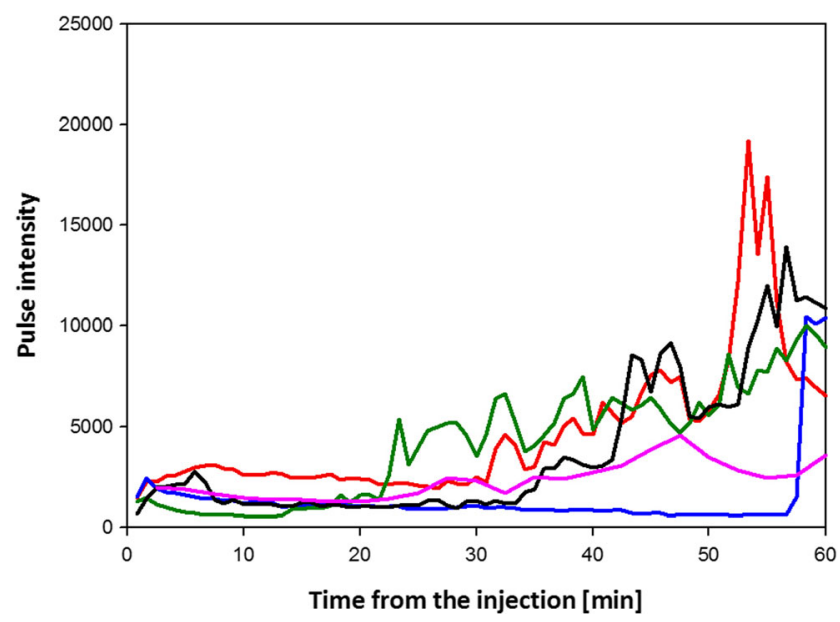

Fig. 1 Time-activity curves for five study subjects with bile tracer activity in the gastric tube during scintigraphy. Time from tracer injection is on $x$-axis and recorded tracer activity (pulse intensity) in the gastric tube on $y$-axis

Hepatobiliary scintigraphy was performed for all patients at 10.7 months (8.6-13.0) after the surgery. Five patients had BR into the gastric tube during the scan (Fig. 1). Selected images of one representative patient are shown in Fig. 2. Bile tracer was found in the gastric tube as early as $23 \mathrm{~min}$ from the tracer injection for one patient (range 23-58 $\mathrm{min}$ ) and maximum activity was observed at $58 \mathrm{~min}$ (Fig. 1). Highest concentration of bile tracer (BRi) in the gastric tube during the scan was $8 \%(1-8 \%)$ of maximal activity. Concentration of bile tracer in the gastric tube was diminishing at the end of the 60-min scan, but total resolution of bile tracer was not observed. There was no sign of bile reflux into the esophagus in any of the SPECT/ CT scans.

GerdQ scores of two patients were above 8, which correlates with reflux disease. One of them had BR into the gastric tube in scintigraphy. Neither of them had BR into the esophagus.

An additional gastroscopy was done for all patients with a sign of BR in hepatobiliary scintigraphy. Four patients were invited for a gastroscopy at 14.4 months (12.4-16.7). Three of them had a normal endoscopic finding as well as normal histology in biopsies. One patient had mild esophagitis in the endoscopic assessment and similar finding in biopsies from the Z-line. He had no reflux symptoms (GerdQ score 5), and there was no evidence of BR into the esophagus in
Fig. 2 Dynamic scan of one representative patient. Frame number is $\mathrm{x}-\mathrm{y}$ followed by time from injection of bile tracer

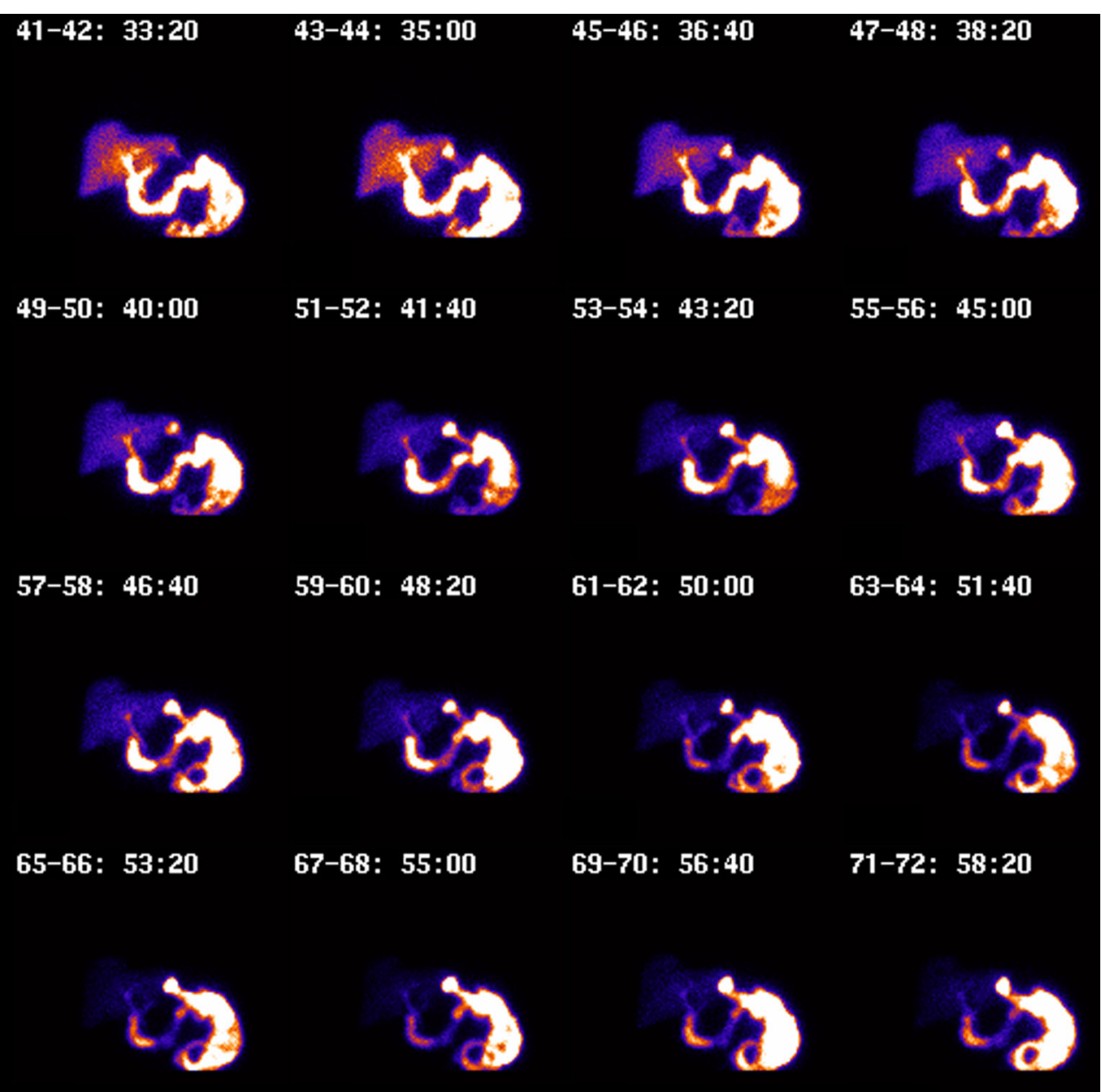


scintigraphy nor in the endoscopic assessment. Prior to surgery, he had similar findings in gastroscopy. One patient had a gastroscopy prior to scintigraphy at 10.1 months after the operation due to malabsorption and reflux symptoms. He had GerdQ score 12, which is considered significant for reflux disease. His endoscopic assessment and biopsies showed gastritis without HP infection but no esophagitis. Prior to MGB, he had normal endoscopy findings with normal biopsies. A 24-h impedance and $\mathrm{pH}$ measurement of the esophagus and gastric tube showed a continuous non-acid reflux. At 12 months, he had iron deficiency and hypoalbuminemia requiring intravenous administration of iron as well as dietary supplementation of protein. Due to difficult reflux symptoms and malabsorption, he underwent revision surgery in which MGB was converted to Roux-en-Y gastric bypass (RYGB) with $60 \mathrm{~cm}$ biliary and $120 \mathrm{~cm}$ alimentary limbs.

One other patient without any sign of BR in the scintigraphy reported heartburn and reflux symptoms and had GerdQ score 11. She was diagnosed with a marginal ulcer, which healed with proton pump inhibitor. Another patient with a negative scintigraphy reported difficulties in swallowing. He was diagnosed with intestinal metaplasia at the gastroesophageal junction in gastroscopy at 9 months after the surgery. Prior to surgery, he had a similar macroscopic finding in gastroscopy but no biopsies were obtained. A 24-h esophageal $\mathrm{pH}$ measurement revealed a difficult night-time acid reflux.

\section{Conclusion}

MGB technique was developed as a modification of the Mason's loop gastric bypass in which a transverse gastric pouch was divided at the fundus. McCarthy et al. reported endoscopic evidence of gastritis in $71 \%$ and high concentrations of bile acids in the gastric pouch after Mason's loop procedure in 28 randomly selected patients after mason's loop gastric bypass in 1985 [13]. It has been stated the modification of the gastric pouch has diminished the bilious contents in the gastric tube and esophagus but the exposure of ventricular and esophageal mucosa to bile and the clinical relevance of it are not yet disclosed [14].

In a retrospective analysis of five centers, the main indication for revision surgery after MGB was BR [6]. Two large studies with 1000 patients reported a re-operation rate of 0.4 and $0.7 \%$ due to BR after MGB. However, completeness of follow-up was not reported in these studies [2, 14]. Data on non-symptomatic BR after MGB has not been published. High levels of BR have been found in non-obese patients with Barrett's esophagus in two previous studies [15, 16]. Bile reflux gastritis has also been found to associate with intestinal metaplasia at the cardia [17]. On the other hand, in another study, the risk for Barrett's esophagus was not significantly related to higher concentrations of bile acids in the stomach
[18]. The role of bile acids as a risk factor for gastric carcinoma has been a subject of discussion, especially in conjunction with H. pylori (HP) colonization [19]. In fact, BR has been reported to cause more severe mucosal lesions in patients with chronic gastritis, especially in conjunction with HP [20].

BR has been stated to cause acute and chronic inflammatory changes in the gastric mucosa along with histological changes in gastro-esophageal junction, which eventually may lead to Barrett's esophagus [21]. According to a systematic review by McQuaid et al., bile acids have been found to induce esophageal squamous cells to resemble intestinal-type cells and to promote Barrett's cells to increase intestinal type genes in in vitro studies [22]. However, Nason et al. did not find similar results in vivo [18]. Park et al. showed that bile acids induce COX-2 expression in gastric cells to generate intestinal metaplasia and predispose to neoplastic changes in the gastric mucosa [23].

$\mathrm{BR}$ in the stomach is also considered a physiologic phenomenon. According to the Society of Nuclear Medicine guidelines for hepatobiliary scintigraphy, activity may reflux from the duodenum into the stomach but a marked BR in a symptomatic patient correlates with bile gastritis [12]. Previous scintigraphic studies of healthy unoperated controls by Tolin et al. and Mackie et al. have shown a BR into the stomach with mean BRi 10 and 8\%, respectively [24, 25]. Chen et al. compared hepatobiliary scintigraphies of patients with a known duodenogastric reflux disease with healthy controls. They found significantly higher amounts of bile tracer in the ventricles of patients with a known duodenogastric reflux disease with BRi up to $28 \%$. Also in this study, all healthy controls but one had evidence of BR with BRi 1-9\% [9]. In our series, the highest amount of bile tracer in the gastric tube after MGB was $8 \%$, which is similar to the finding of healthy subjects in these previous scintigraphic studies.

Tolone et al. studied high resolution manometries after omega-loop gastric bypass. They discovered that intragastric pressures and gastroesophageal pressure gradient statistically diminished after the surgery. They concluded that this would result in a decreased incidence of gastroesophageal reflux after omega-loop gastric bypass [26].

A review by Mahawar et al. addressed the issue of $B R$ after MGB [27]. They concluded that according to the literature, BR will lead to higher incidence of histological gastritis, but it does not always translate into adverse symptomatic outcome.

Sundbom et al. studied hepatobiliary scintigraphies after RYGB operations, and they found BR into the excluded stomach in $36 \%$ of patients. The effect of bile might be more harmful for the mucosa of the excluded stomach after RYGB compared to the gastric pouch after MGB due to the fact that bile in the excluded stomach will not be diluted or mixed with normal gastric contents as liquid and solid food are not passing through the excluded ventricle [28].

Risk of gastric cancer has been associated with gastric surgery in previous studies. This has been thought to be a 
consequence of BR induced by gastric surgery [29, 30]. However, later, it has been stated that the increased gastric cancer risk after gastric surgery is most likely because of synergistic effect of BR and HP infection [19]. Therefore, HP eradication prior to gastric bypass surgery is of utmost importance. In a systematic review by Scozzari et al. in 2013, 33 cases of carcinoma after bariatric surgery were reported [31]. To date, only one case of gastric carcinoma after MGB has been published [32]. It is noteworthy that all cases of gastric carcinoma after gastric bypass operations have been in the bypassed stomach. The role of BR in the carcinogenesis in the bypassed stomach is unclear and needs further investigation.

BR after MGB is a subject of ongoing debate, even though until now there has not been a study that actually demonstrated BR after MGB. Everyone agrees that bile exposure to esophageal mucosa is extremely harmful. The effect of bile exposure to the gastric mucosa on the other hand is more controversial. Our case series demonstrates a modest BR in hepatobiliary scintigraphy after MGB in five out of nine consecutive patients. However, none of the patients had any sign of bile reflux into the esophagus. The amount of bile in the gastric tube was fairly small but the entire duration of BR could not be determined by this method. Only one patient with recorded BR had reflux symptoms as well as histologic gastritis and he required reoperation due to malabsorption and non ulcerative GERD. Gastric biopsies of non-symptomatic patients with a positive scintigraphy did not show foveolar hyperplasia or other inflammatory changes in the gastric tube. This is either due to the fact that the amount of bile in the gastric tube is not enough to cause histologic changes or it may also be due to a fairly short time between surgery and follow-up gastroscopy. Two patients had reflux symptoms with a negative BR finding in scintigraphy. All things considered, scintigraphies did not correlate well with clinical findings of bile reflux.

Our study is limited due to small number of participants and lack of a control group. The imaging protocol was not originally designed for the detection of bile reflux after bariatric surgery and therefore it has some limitations: We used our routine protocol that included an anterior $60 \mathrm{~min}$ dynamic scan of the thorax and abdomen. It is possible that some transient late esophageal BR peaks were missed. Also, our protocol does not tell us whether BR is present beyond the $60 \mathrm{~min}$ scan. An intermittent scan during a longer period of time would provide more information.

This is the first report on hepatobiliary scintigraphies after MGB. Our results indicate that transient bile reflux is common after MGB in the gastric tube, but not in the esophagus. The clinical relevance of bile reflux needs further studies.

\section{Compliance with Ethical Standards}

Conflict of Interest The authors declare that they have no conflict of interest.
Statement of Informed Consent Informed consent was obtained from all individual participants included in the study.

Ethical Approval This study was approved by the surgical ethics committee of our institution.

Sources of Funding This study was funded by Finnish governmental research funds (EVO).

\section{References}

1. Rutledge R. The mini-gastric bypass: experience with the first 1 , 274 cases. Obesity Surg. 2001;11(3):276-80.

2. Noun R, Skaff J, Riachi E, et al. One thousand consecutive minigastric bypass: short- and long-term outcome. Obesity Surg. 2012;22(5):697-703

3. Lee W, Yu P, Wang W, et al. Laparoscopic Roux-en-Y versus minigastric bypass for the treatment of morbid obesity: a prospective randomized controlled clinical trial. Ann Surg. 2005;242(1):20-8.

4. Lee WJ, Ser KH, Lee YC, et al. Laparoscopic Roux-en-Y vs. minigastric bypass for the treatment of morbid obesity: a 10-year experience. Obesity Surg. 2012;22(12):1827-34.

5. Victorzon M. Single-anastomosis gastric bypass: better, faster, and safer? Scand J Surg. 2015;104(1):48-53.

6. Johnson WH, Fernanadez AZ, Farrell TM, et al. Surgical revision of loop ("mini") gastric bypass procedure: multicenter review of complications and conversions to Roux-en-Y gastric bypass. Surgery for Obesity \& Related Diseases. 2007;3(1):37-41.

7. Stein HJ, Smyrk TC, DeMeester TR, et al. Clinical value of endoscopy and histology in the diagnosis of duodenogastric reflux disease. Surgery. 1992; discussion 803-4112(4):796-803.

8. Mittal BR, Ibrarullah M, Agarwal DK, et al. Comparative evaluation of scintigraphy and upper gastrointestinal tract endoscopy for detection of duodenogastric reflux. Ann Nucl Med. 1994;8(3):1836.

9. Chen TF, Yadav PK, Wu RJ, et al. Comparative evaluation of intragastric bile acids and hepatobiliary scintigraphy in the diagnosis of duodenogastric reflux. World J Gastroenterol. 2013;19(14): 2187-96.

10. Yumuk V, Tsigos C, Fried M, et al. European guidelines for obesity management in adults. Obesity Facts. 2015;8(6):402-24.

11. Jones R, Junghard O, Dent J, et al. Development of the GerdQ, a tool for the diagnosis and management of gastro-oesophageal reflux disease in primary care. Aliment Pharmacol Ther. 2009;30(10): 1030-8.

12. Tulchinsky M, Ciak BW, Delbeke D, et al. SNM practice guideline for hepatobiliary scintigraphy 4.0. J Nucl Med Technol. 2010;38(4): 210-8.

13. McCarthy HB, Rucker RDJ, Chan EK, et al. Gastritis after gastric bypass surgery. Surgery. 1985;98(1):68-71.

14. Chevallier JM, Arman GA, Guenzi M, et al. One thousand single anastomosis (omega loop) gastric bypasses to treat morbid obesity in a 7-year period: outcomes show few complications and good efficacy. Obesity Surg. 2015;25(6):951-8.

15. Iftikhar SY, Ledingham $\mathrm{S}$, Steele RJ, et al. Bile reflux in columnar-lined Barrett's oesophagus. Ann R Coll Surg Engl. 1993;75(6):411-6.

16. Martinez de Haro L, Ortiz A, Parrilla P, et al. Intestinal metaplasia in patients with columnar lined esophagus is associated with high levels of duodenogastroesophageal reflux. Ann Surg. 2001;233(1):34-8.

17. Dixon MF, Mapstone NP, Neville PM, et al. Bile reflux gastritis and intestinal metaplasia at the cardia. Gut. 2002;51(3):351-5. 
18. Nason KS, Farrow DC, Haigh G, et al. Gastric fluid bile concentrations and risk of Barrett's esophagus. Interactive Cardiovascular \& Thoracic Surgery. 2007;6(3):304-7.

19. Li XB, Lu H, Chen HM, et al. Role of bile reflux and Helicobacter pylori infection on inflammation of gastric remnant after distal gastrectomy. J Dig Dis. 2008;9(4):208-12.

20. Chen S, Mo J, Cao Z, et al. Effects of bile reflux on gastric mucosal lesions in patients with dyspepsia or chronic gastritis. World $\mathrm{J}$ Gastroenterol. 2005;11(18):2834-7.

21. Dixon MF, Neville PM, Mapstone NP, et al. Bile reflux gastritis and Barrett's oesophagus: further evidence of a role for duodenogastrooesophageal reflux? Gut. 2001;49(3):359-63.

22. McQuaid KR, Laine L, Fennerty MB, et al. Systematic review: the role of bile acids in the pathogenesis of gastro-oesophageal reflux disease and related neoplasia. Aliment Pharmacol Ther. 2011;34(2):146-65.

23. Park MJ, Kim KH, Kim HY, et al. Bile acid induces expression of COX-2 through the homeodomain transcription factor CDX1 and orphan nuclear receptor SHP in human gastric cancer cells. Carcinogenesis. 2008;29(12):2385-93.

24. Tolin RD, Malmud LS, Stelzer F, et al. Enterogastric reflux in normal subjects and patients with Bilroth II gastroenterostomy. Measurement of enterogastric reflux. Gastroenterology. 1979;77(5):1027-33.

25. Mackie C, Hulks G, Cuschieri A. Enterogastric reflux and gastric clearance of refluxate in normal subjects and in patients with and without bile vomiting following peptic ulcer surgery. Ann Surg. 1986;204(5):537-42.

26. Tolone S, Cristiano S, Savarino E, et al. Effects of omega-loop bypass on esophagogastric junction function. Surg Obes Relat Dis. 2016;12(1):62-9.

27. Mahawar KK, Carr WR, Balupuri S, et al. Controversy surrounding 'mini' gastric bypass. Obes Surg. 2014;24(2):324-33.

28. Sundbom M, Hedenstrom H, Gustavsson S. Duodenogastric bile reflux after gastric bypass: a cholescintigraphic study. Digestive Diseases \& Sciences. 2002;47(8):1891-6.

29. Schafer LW, Larson DE. Melton LJ3, Higgins JA, Ilstrup DM. The risk of gastric carcinoma after surgical treatment for benign ulcer disease. A population-based study in Olmsted County, Minnesota. N Engl J Med. 1983;309(20):1210-3.

30. Viste A, Bjornestad E, Opheim P, et al. Risk of carcinoma following gastric operations for benign disease. A historical cohort study of 3470 patients. Lancet. 1986;2(8505):502-5.

31. Scozzari G, Trapani R, Toppino M, et al. Esophagogastric cancer after bariatric surgery: systematic review of the literature. Surgery for Obesity \& Related Diseases. 2013;9(1):133-42.

32. Wu C, Lee W, Ser K, et al. Gastric cancer after mini-gastric bypass surgery: a case report and literature review. Asian Journal of Endoscopic Surgery. 2013;6(4):303-6. 